\title{
Anti-Osteoporotic Effect of Soy Isoflavones Intake on Low Bone Mineral Density Caused by Voluntary Exercise and Food Restriction in Mature Female Rats
}

\author{
Kaoru YANAKA ${ }^{1,2}$, Mitsuru HiguCHI ${ }^{3}$ and Yoshiko IsHIMI ${ }^{4}$ \\ ${ }^{1}$ Graduate School of Sport Sciences, Waseda University, 2-579-15 Mikajima, \\ Tokorozawa, Saitama 359-1192, Japan \\ ${ }^{2}$ Kushiro Sanjikai Hospital, 4-30 Nusamai-cho, Kushiro, Hokkaido 085-0836, Japan \\ ${ }^{3}$ Faculty of Sport Sciences, Waseda University, 2-579-15 Mikajima, \\ Tokorozawa, Saitama 359-1192, Japan \\ ${ }^{4}$ National Institutes of Biochemical Innovation, Health and Nutrition, 1-23-1 Toyama, \\ Shinjuku-ku, Tokyo 162-8636, Japan \\ (Received January 24, 2019)
}

\begin{abstract}
Summary Female athlete triad (FAT) is an interrelationship between menstrual dysfunction, low energy availability with or without eating disorder, and decreased bone mineral density (BMD) in female athletes. The purpose of this study was to investigate whether isoflavone intake can prevent bone loss caused by voluntary wheel running under energyrestricted condition. We used a female rat model of osteoporosis for female athletes established previously. Fourteen female Sprague-Dawley rats (8-wk old) were fed ad libitum and had free access to wheels throughout the study. At $18 \mathrm{wk}$ of age, the rats were divided randomly into the following groups: 1) running control (RC), 2) running energy restriction (RR), and 3) running energy restriction and isoflavone-fed ( $R R+I s o$ ) groups. The $R R$ group was 30\% dietary restricted. The RR + Iso group was 30\% dietary restricted and fed the diet containing $0.5 \%$ isoflavone powder (Fujiflavone P40). The experimental period lasted $31 \mathrm{wk}$. At the end of this experiment, BMD of the proximal femur in the RR group was significantly lower than that in the RC group. However, the BMD in the RR + Iso group was not significantly different from that in the RC group. Moreover, the plasma estradiol $\left(\mathrm{E}_{2}\right)$ level in the RR and RR+Iso groups was significantly lower than that in the RC group. These findings suggest that isoflavone intake inhibited bone loss when the $\mathrm{E}_{2}$ level was low in female mature rat model. Our findings may reveal the possible novel role of isoflavone in osteoporosis among female athletes.
\end{abstract}

Key Words female athlete, osteoporosis, estradiol, BFR, femur

Female athlete triad (FAT) published in 1997 by the American College of Sports Medicine Position Stand (1), is a syndrome that is an agglomeration of eating disorder, amenorrhea, and osteoporosis, although these three symptoms may not be present simultaneously.

Osteoporosis in female athletes occurs when there is inadequate energy availability with or without menstrual dysfunction. The importance of adequate energy intake for bone metabolism is well established. Low energy availability negatively affects the bones, leading to rapid increase in bone resorption and decline in bone formation directly related to the severity of energy availability (2). It cannot be the only factor; estrogen status is thought to be a major factor of osteoporosis in female athletes. This is evidenced by the fact that it occurs in athletes with hypothalamic amenorrhea (3). A previous study has reported that the luteinizing hormone (LH) level is suppressed by low energy availability and strenuous exercise (4). Our previous study also showed that the LH level decreased with exercise and diet restrictions (5).

E-mail:nut_yanaka@yahoo.co.jp
Soy isoflavones have structural similarities to that of estradiol $\left(\mathrm{E}_{2}\right)$, exhibiting week estrogenic actions by binding to the estrogen receptors (ERs). There are 15 kinds of isoflavone isomers (6). The ability of isoflavones to bind to the ER is higher for genistein and daidzein than for other compounds (7). Numerous studies have elucidated that isoflavone intake can prevent bone loss in postmenopausal women and ovariectomized animals $(8-12)$. Its action mechanism involves the inhibition of bone resorption and bone formation.

Studies have demonstrated that running exercise and food reduction induce low bone strength and bone mass in mature (5) and young female rats $(13,14)$.

Our previous study showed that the low bone mineral density (BMD) level of the femur caused by energy restriction and voluntary wheel running reduced the reproduction hormone levels in mature rats. However, the efficacy of long-term isoflavone intake on BMD, bone histomorphometry, and female hormone levels is unclear. The effect of isoflavone intake on BMD, bone mineral contents (BMCs), and reproductive hormonal level can be evaluated using an FAT animal model (5). In this 
Table 1. Composition of the experimental diets.

\begin{tabular}{lccc}
\hline \multirow{2}{*}{ Ingredient } & \multicolumn{2}{c}{ Diet } \\
\cline { 2 - 4 } & AIN-93G & AIN-93M & 0.5\% Isoflavone contained diet \\
\hline & & & g/kg diet \\
Cornstarch & 529.4 & 620.7 & 620.7 \\
Casein & 200 & 140.0 & 140.0 \\
Sucrose & 100 & 100.0 & 95.0 \\
Corn oil & 70 & 40.0 & 40.0 \\
Cellulose & 50 & 50.0 & 50.0 \\
Mineral mix & 35 & 35.0 & 35.0 \\
Vitamin mix (AIN-93-VX) & 10 & 10.0 & 10.0 \\
L-Cystine & 3 & 1.8 & 2.5 \\
Choline bitartrate & 2.5 & 2.5 & 0.008 \\
tert-Butylhydroquione & 0.14 & 0.008 & 5.0 \\
Fujiflavone (P40) & - & - & 1,000 \\
\hline
\end{tabular}

Table 2. Isoflavone constituent and contents in Fujiflavone $\mathrm{P} 40$.

\begin{tabular}{lc}
\hline & Content, $\%[\mathrm{w} / \mathrm{w}]$ \\
\hline Daidzin & 22.48 \\
Glycitin & 14.00 \\
Genistin & 5.47 \\
6"-O-Malonyldaizin & 0.22 \\
6"-O-Malonylglycitin & 0.19 \\
6"-O-Malonylgenistin & 0.00 \\
6"-O-Acetyldaidzin & 2.26 \\
6"-O-Acetylglycitin & 1.30 \\
6"-O-Acetylgenistin & 0.67 \\
Daidzain & 0.26 \\
Glycitain & 0.51 \\
Genistein & 0.07 \\
Total isoflavone & 47.34 \\
(Aglycone equivalent) & 29.52 \\
\hline
\end{tabular}

study, we examined the effect of isoflavone intake on bone loss caused by energy restriction with voluntary wheel running in female mature rats.

\section{MATERIALS AND METHODS}

Experimental protocol. Sixteen female Sprague-Dawley rats (8-wk old) were purchased from the Shizuoka Laboratory Animal Center (Shizuoka, Japan). The rats were housed in individual cages in a temperature and humidity controlled room $\left(23 \pm 1^{\circ} \mathrm{C}\right.$ and $60 \pm 5 \%$ relative humidity) with a 12-h light/dark cycle. The rats had free access to the wheels. The rats were fed the diet described in Table 1. Food and water were available to all the rats ad libitum during the initial 10-wk baseline period. When the rats were 18-wk old, they were randomly divided into three groups, running control (RC) group, running and 30\% food restriction (RR) group, and running and 30\% food restriction with $0.5 \%$ isofla- vone (RR+Iso) group. Diet intake in the RR and RR + Iso groups was calculated from their average food intake for $2 \mathrm{wk}$ before the restriction period. The rats were fed an AIN-93G or AIN-93M diet with corn oil instead of soybean oil during the baseline or restriction period, respectively. Fujiflavone $\mathrm{P} 40$ is a soybean seed isoflavone product (Fujicco Co. Ltd., Kobe Japan), which was used in this study. Its composition is shown in Table 2. Body weight and food intake were measured every third or fourth day during the initial baseline period and every second day during the restriction period. Running distance was recorded five times per week. After $23 \mathrm{wk}$, the estrous cycle was monitored by daily vaginal smear pattern for $2 \mathrm{wk}$ before dissection. The vaginal smear patterns were recorded as follows: proestrus, estrus, metestrus, and diestrus. If the vaginal smears indicated the metestrus or diestrus stage, the rats were subcutaneously anesthetized and decapitated. Blood was collected by cardiac puncture exsanguination. Plasma sample was collected by centrifuging the blood sample at $1,942 \times g$ for $15 \mathrm{~min}$ and stored at $-80^{\circ} \mathrm{C}$ until further analysis. The right femora were removed to measure the BMD and bone BMC. The distal region of the left femora was analyzed to evaluate the effect on bone morphology. All procedures involving animals were in accordance with the Guidelines for the care and use of laboratory animals by the National Institute of Health and Nutrition (Tokyo, Japan) and the ethics committee approved the study protocol (1211) in 2012.

Measurement of BMD. Bone mineral density of the right femur of each rat was measured by dual-energy X-ray absorptiometry (DXA) using a densitometer adapted for animal research (model DCS-600R; Hitachi Aloka, Tokyo, Japan). The BMD was calculated using the $\mathrm{BMC}$ of the measured area. The BMD of three regions of the femur, namely proximal femur, middle femur, and distal femur, was accessed to determine regional differences in the femur.

Bone histomorphometric analysis. Bone labeling by 

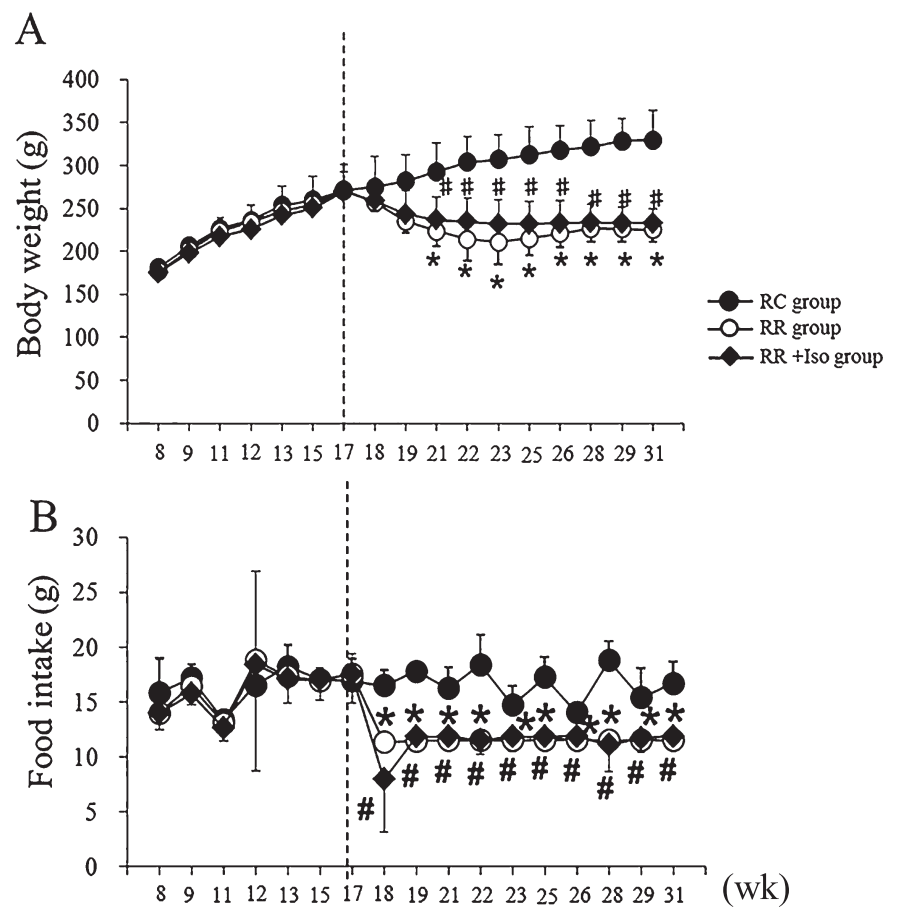

Fig. 1. Time course of change in (A) body weight, and (B) food intake during the experimental period in the wheel running with ad libitum-feeding $(\mathrm{RC})$ group, wheel running with restricted-feeding $(\mathrm{RR})$ group, and running with restrictionisoflavone-feeding (RR+Iso) group. Values are given as means \pm SD; $n=4$ for the RC group, $n=6$ for the RR group, $n=6$ for the RR+Iso group. ${ }^{*} p<0.05$, RC vs. RR group; ${ }^{*} p<0.05$, RC vs. RR+Iso group, by one-way ANOVA with repeated measures over time.

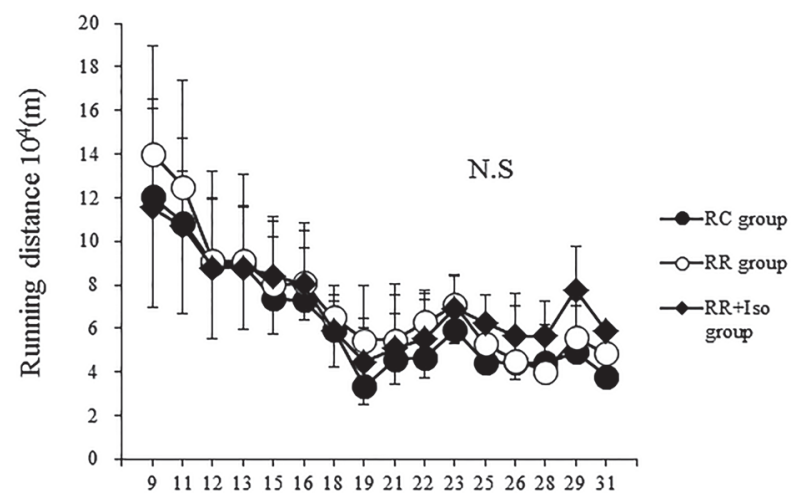

Fig. 2. Running distance for rats included in the wheelrunning groups throughout of the study. (๑) running ad libitum-fed (RC) group, $(\bigcirc)$ running restricted-fed $(\mathrm{RR})$ group, and $(\bullet)$ running restricted-fed isoflavone (RR + Iso) group throughout the total duration of the experiment. Values are given as means \pm SD; $n=4$ for the RC group, $n=6$ for the RR group, $n=6$ for the $\mathrm{RR}+$ Iso group, by one-way ANOVA.

subcutaneous injection of tetracycline and calcein at $20 \mathrm{mg} / \mathrm{kg}^{-1}$ body weight (bw) was performed on day 5 or 1 before $23 \mathrm{wk}$. The left femora were fixed in $70 \%$ ethanol, and the undecalcified bone was embedded in methyl methacrylate. A 5- $\mu$ m-thick portion was cut longitudinally in the distal region of the femora and sectioned. The analysis was performed using the semiautomatic image analyzing system connected to a light microscope. The following parameters were evaluated: bone volume/total volume/tissue volume (BV/TV, \%),
A

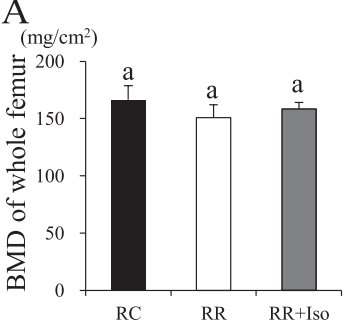

$\mathrm{B}$

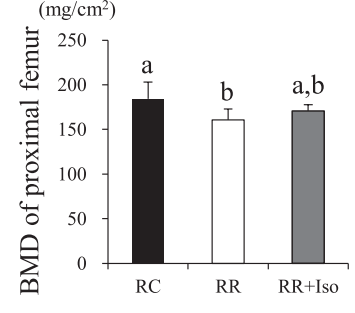

$\mathrm{C}$

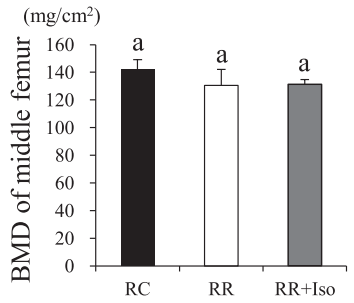

$\mathrm{D}$

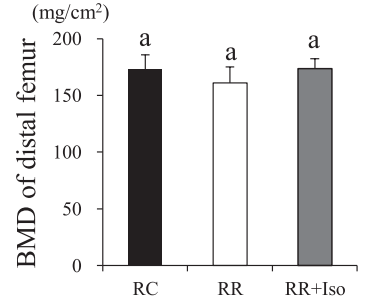

Fig. 3. Effects of energy restriction with wheel running and isoflavone intake for $18 \mathrm{wk}$ on BMD in female rats. Whole femur BMD (A), proximal BMD (B), middle BMD (C), distal BMD (D). Values are means \pm SD, $n=4-6$. Means with different letters differ, $p<0.05$ by one-way ANOVA.

trabecular thickness (Tb.Th, $\mu \mathrm{m}$ ), osteoid surface/bone surface (OS/BS, \%), osteoblast surface/bone surface (Ob.S/BS, \%), number of osteoblast/bone surface (N.Ob/ BS, N/mm), number of osteoclast/bone surface (N.Oc/ $\mathrm{BS}, \mathrm{N} / \mathrm{mm}$ ), mineral absorption rate (MAR, $\mu \mathrm{m} / \mathrm{d}$ ), bone 

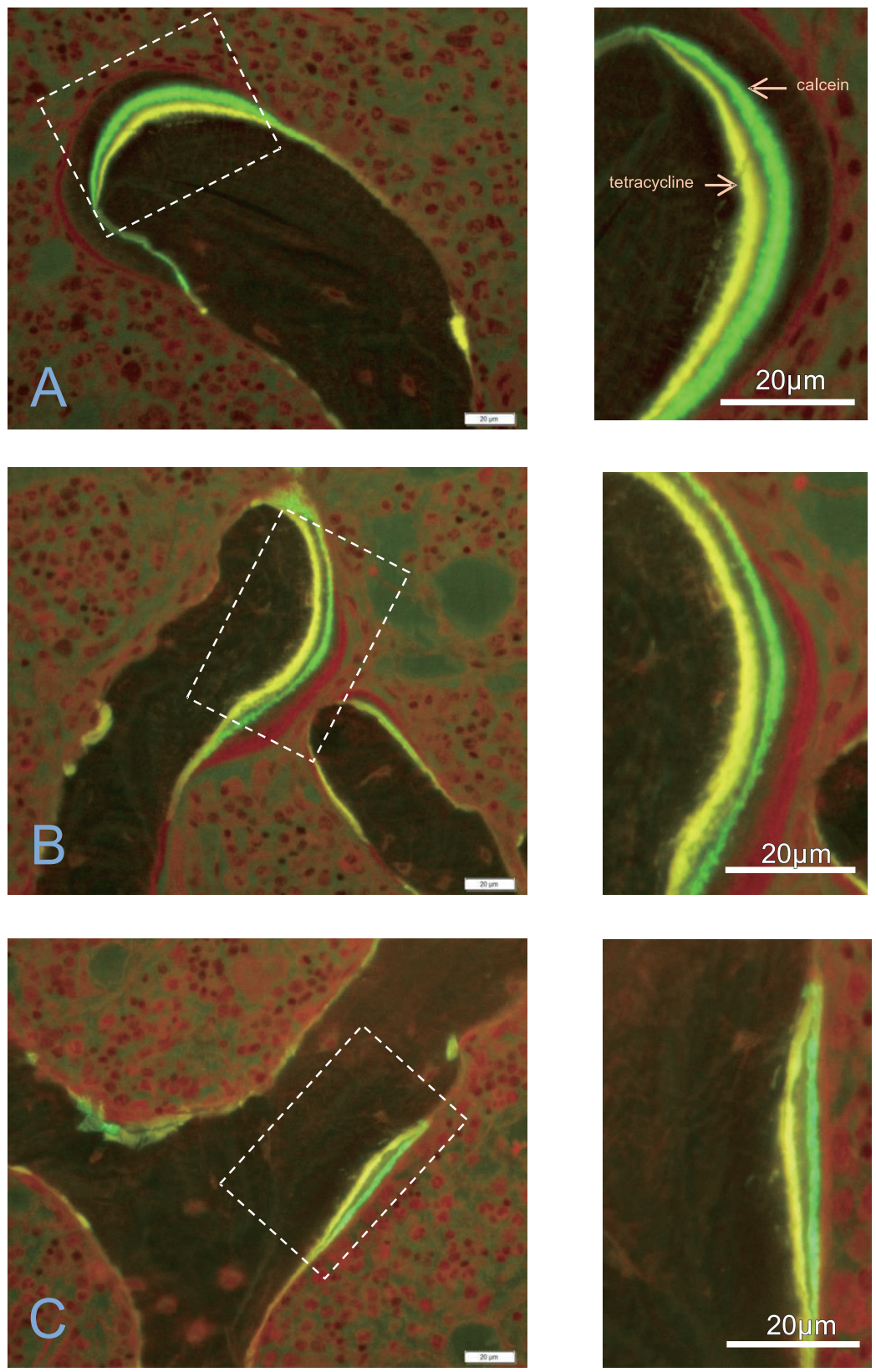

Fig. 4. Photomicrograph of the distal femur of each group: (A) RC group, (B) RR group, (C) RR+Iso group. It was double labeled with yellow label tetracycline and yellow green label calcein.

formation rate/bone surface (BFR/BS $\mathrm{mm}^{2} / \mathrm{mm}^{2} / \mathrm{y}$ ), bone formation rate/bone volume (BFR/BV, \% $\mathrm{y})$, bone formation rate/tissue volume (BFR/TV, \% y), trabecular number (Tb.N, N/mm), and trabecular space (Tb.Sp, $\mu \mathrm{m})$. The histomorphometric analysis was performed by the Ito Bone Histomorphometry Institute (Niigata, Japan).

Plasma biochemical analysis. The levels of $\mathrm{E}_{2}$ and $\mathrm{LH}$ were measured using enzyme-linked immunosorbent assay kits (Estradiol sensitive ELISA DRG, Springfield, NJ; Rodent LH, Newark, CA).

Statistical analyses. The data are presented as mean \pm standard deviation (SD). The effects of energy restriction on body weight and food intake were analyzed using the one-way analysis of variance (ANOVA) with repeated measures. The one-way ANOVA with
Tukey's post hoc test was performed on running distance, femoral BMC, BMD, and each parameter in the histomorphometric and plasma biochemical analyses. All statistical analyses were conducted using SPSS Statistical Packages (Ver. 21.0, SPSS Inc., Chicago, IL).

\section{RESULTS}

Body weight, food intake, and running distance

We examined the influence of isoflavone intake on the $\mathrm{BMD}$ and $\mathrm{E}_{2}$ level in energy-restricted mature female rats. Food restriction was started when the rats were 18-wk old. The body weight of the rats gradually increased in all the groups until food restriction. The body weight of the rats was significantly lower $(p<0.05)$ in the RR and $\mathrm{RR}+$ Iso groups than in the RC group $(p<0.05)$ when the rats were 21-31-wk old (Fig. 1A). Food intake was 
Table 3. Bone histomorphometry of cancellous bone of the distal femur in rats.

\begin{tabular}{|c|c|c|c|c|}
\hline Bone variable & $\mathrm{RC}$ & $\mathrm{RR}$ & $\mathrm{RR}+\mathrm{Iso}$ & $p$ value \\
\hline $\mathrm{BV} / \mathrm{TV}, \%$ & $33.5 \pm 4.3^{\mathrm{a}}$ & $19.1 \pm 8.5^{b}$ & $21.4 \pm 5.5^{\mathrm{b}}$ & $<0.05$ \\
\hline Tb.Th, $\mu \mathrm{m}$ & $70.8 \pm 9.9^{a}$ & $58.5 \pm 8.8^{\mathrm{a}, \mathrm{b}}$ & $55.4 \pm 5.2^{\mathrm{b}}$ & $<0.05$ \\
\hline OS/BS, \% & $3.7 \pm 1.9$ & $9.7 \pm 5.9$ & $3.0 \pm 3.1$ & N.S \\
\hline Ob.S/BS, \% & $3.7 \pm 1.1$ & $10.3 \pm 6.9$ & $2.6 \pm 2.5$ & N.S \\
\hline $\mathrm{N} . \mathrm{Ob} / \mathrm{BS}, \mathrm{N} / \mathrm{mm}$ & $3.0 \pm 1.1$ & $7.4 \pm 4.7$ & $2.0 \pm 1.9$ & N.S \\
\hline N.Oc/BS, N/mm & $3.0 \pm 1.8$ & $2.2 \pm 1.8$ & $1.6 \pm 0.5$ & N.S \\
\hline $\mathrm{MAR}, \mu \mathrm{m} / \mathrm{d}$ & $1.3 \pm 0.1^{\mathrm{a}}$ & $1.2 \pm 0.1^{\mathrm{a}}$ & $0.9 \pm 0.2^{b}$ & $<0.05$ \\
\hline $\mathrm{BFR} / \mathrm{BS}, \mathrm{mm}^{2} / \mathrm{mm}^{2} / \mathrm{y}$ & $0.065 \pm 0.026^{\mathrm{a}, \mathrm{b}}$ & $0.084 \pm 0.052^{\mathrm{a}}$ & $0.019 \pm 0.01^{b}$ & $<0.05$ \\
\hline $\mathrm{BFR} / \mathrm{BV}, \% \mathrm{y}$ & $178.9 \pm 45.6^{\mathrm{a}, \mathrm{b}}$ & $311.9 \pm 218.8^{\mathrm{a}}$ & $67.1 \pm 36.5^{b}$ & $<0.05$ \\
\hline BFR/TV, \% y & $60.6 \pm 20.8^{\mathrm{a}}$ & $45.8 \pm 21.2^{\mathrm{a}}$ & $15.2 \pm 8.5^{\mathrm{b}}$ & $<0.05$ \\
\hline Tb.N, N/mm & $4.8 \pm 0.7$ & $3.2 \pm 1.3$ & $3.8 \pm 0.7$ & N.S \\
\hline Tb.Sp, $\mu \mathrm{m}$ & $142.2 \pm 26.7$ & $318.0 \pm 210.3$ & $214.8 \pm 60.6$ & N.S \\
\hline
\end{tabular}

Values means \pm SD, $n=3$, means with different letters differ, $p<0.05$ by one-way ANOVA.

Table 4. Plasma concentrations of $\mathrm{E}_{2}$ and $\mathrm{LH}$ in rats. Effects of energy restriction with wheel running and isoflavone intake on plasma $\mathrm{E}_{2}$ and $\mathrm{LH}$ concentration.

\begin{tabular}{lcccc}
\hline & $\mathrm{RC}$ & $\mathrm{RR}$ & $\mathrm{RR}+$ Iso & $p$ value \\
\hline $\mathrm{E}_{2}(\mathrm{pmol} / \mathrm{L})$ & $26.0 \pm 6.3^{\mathrm{a}}$ & $18.5 \pm 1.8^{\mathrm{b}}$ & $16.9 \pm 1.5^{\mathrm{b}}$ & $<0.05$ \\
$\mathrm{LH}(\mathrm{ng} / \mathrm{mL})$ & $3.1 \pm 1.4$ & $2.5 \pm 2.7$ & $1.6 \pm 0.5$ & N.S \\
\hline
\end{tabular}

Values means \pm SD, $n=4-6$, means with different letters differ, $p<0.05$ by one-way ANOVA.

significantly lower in the RR and $\mathrm{RR}+$ Iso groups than in the RC group $(p<0.05)$ when the rats were $18-31 \mathrm{wk}$ old (Fig. 1B). Running distance was not significantly different among the groups throughout the study (Fig. 2). Femoral BMD measurement

We evaluated the effect of isoflavone intake on low BMD caused by energy restriction with voluntary wheel running in female mature rats. The proximal BMD was lower in the RR group than in the RC group $(p<0.05)$. However, no difference was observed between the RC and RR+Iso groups (Fig. 3B). The BMD of the whole femur was lower in the RR group than in the RC group $(p=0.067)$. However, there was no difference between the RC and RR+Iso groups (Fig. 3A). Furthermore, there was no difference in bone area among the groups in the three regions of the femur.

\section{Histomorphometric analysis of the femur}

The BV/TV in the RR and RR+Iso groups was significantly lower than that in the $\mathrm{RC}$ group $(p<0.05)$. The Tb.Th was significantly lower in the RR + Iso group than in the RC group $(p<0.05)$. The MAR in the $\mathrm{RR}+$ Iso group was significantly lower than that in the $\mathrm{RC}$ and RR groups $(p<0.05)$. The BFR/BS in the $\mathrm{RR}+$ Iso group was lower than that in the RR group $(p<0.05)$. Furthermore, there was no difference between the RR+Iso and $\mathrm{RC}$ groups. The BFR/BV in the $\mathrm{RR}+$ Iso group was lower than that in the RR group $(p<0.05)$, and there was no difference between the RR + Iso and RC groups. The inhibition of bone formation was determined by the thickness of tetracycline and calcein labeling (Fig.
$4 \mathrm{C})$. The BFR/TV in the RR+ Iso group was significantly lower than that in the RC and RR groups $(p<0.05)$. There were no differences in the OS/BS, Ob.S/BS, N.Oc/ BS, Tb.N, and Tb.Sp among the tree groups (Table 3).

Reproduction hormone levels

The $\mathrm{E}_{2}$ level was significantly lower in the RR and $\mathrm{RR}+$ Iso groups than in the RC group. There was no difference in the LH level among the groups (Table 4).

\section{DISCUSSION}

In the present study, we investigated the effect of isoflavone intake on low BMD and low reproduction hormone levels caused by energy restriction with voluntary wheel running in mature female rats. We found that isoflavone intake inhibited bone loss caused by voluntary wheel running and food regimen. Similar to our previous study results, the $\mathrm{E}_{2}$ level was also lower in the $\mathrm{RR}$ or RR + Iso groups than in the RC group. These findings suggest that isoflavone intake inhibited bone loss when the $\mathrm{E}_{2}$ level was low in female athlete rat model.

Previous studies have suggested that isoflavones can exert week estrogen-like effects in estrogen-deficient animal model and postmenopausal women $(9,11,12$, 15-18). Moreover, several studies have shown the positive effects of genistein and daidzein, which have a high affinity for estrogen $\beta$ receptors, as more $\beta$ receptors are localized in bone than $\alpha$ receptors. The effective dose was $0.05-0.18 \%$ for genistein $(15,16)$ and $0.1 \%$ for daidzein (19) in animal diet, respectively. Furthermore, the isoflavone products contain approximately $40 \%$ iso- 
flavones. However, in previous studies they were mixed with $0.22-0.25 \%$ bait to confirm the effect on bone density reduction $(15,19)$. In this study, the isoflavone content in the diet was adjusted to $0.5 \%$ (w/w) using Fujiflavone P40 for isoflavone supply. In the RR+Iso group, the intake amount of total isoflavone aglycon equivalent was $17.4 \mathrm{mg} / \mathrm{d}$. Therefore, the amount of isoflavone intake in this study may be sufficient to evaluate antiosteoporotic effect on bone loss caused by energy restriction with voluntary wheel running.

To obtain the FAT model, it is important to determine whether the estrous cycle is disturbed by energy restriction with voluntary wheel running while considering the experimental technique. When evaluating the estrous cycle, most studies measure and evaluate the plasma $\mathrm{E}_{2}$ and $\mathrm{LH}$ levels $(3,4,20,21)$. We also evaluated the estrous cycle by measuring these reproductive hormone levels (5). On the contrary, Aikawa et al. evaluated the cycle by recording the running distance $(13,14)$. It has been reported that the running cycle is related to the estrous cycle and the running distance becomes longer during the proestrous stage $(22,23)$. In the present study, to confirm the estrous cycle, we also measured the reproductive hormones levels under summer-like conditions. The results revealed that there were several rats whose metestrus period lasted long. In addition, the uterus weight in the RR and RR+Iso groups was significantly lower than that in the RC group (data not shown). From the above results, it is considered that the estrous cycle was disturbed by energy restriction with voluntary wheel running.

A negative energy balance was closely related to the suppression of reproductive functions. Several studies have reported that exercise induced reproductive disturbance is associated with low energy availability (3, 20, 21). In this study, energy availability was not measured however it was considered that the energy availability in the RR and RR+Iso groups was lower than that of RC group. Because the body weight in the RR and $R R+$ Iso groups was lower than that of RC group with the same running distance among the groups. However, the $\mathrm{LH}$ level was not different among the groups in this study (Table 4). This was unlike our previous study results, that is, the LH level was not affected (5). The reason for this might be the time of sampling. Metestrus is when the LH surge ends and returns to the basal level. If sampling is performed at the point when the LH surge occurs, a difference in the LH level might be observed.

In the analysis of DXA, there was no significant difference in the femoral BMD in the proximal region between the RC and RR+Iso groups (Fig. 3); therefore, we believe that isoflavone intake inhibited the decrease in BMD caused by wheel running and food restriction. However, the results of bone morphology analysis were not similar to those of the BMD analysis in this study (Table 3). In particular, the variation in the RR group data was large compared with that in the $\mathrm{RC}$ and $\mathrm{RR}+$ Iso groups. If the number of samples is increased, the results of DXA and bone morphology might be consistent.

In our previous study, we demonstrated bone deterio- ration in wheel running and diet-restricted rats, which was compared with that in sedentary control rats (5). There was no difference in the N.Ob/TV among the groups, the N.Oc/TV was significantly lower in the RR group than in the sedentary control and running control groups. Although it was difficult to interpret the result simply because of the large variation, we considered that it might be the status of reversal phase on bone turnover. In this study, no significant differences in the N.Oc/BS, N.Ob/BS, OS/BS, and Ob.S/BS were observed among the three groups. In addition, there were no significant differences in the Tb.N and Tb.Sp among the groups. On the contrary, the MAR, BFR/BS, and BFR/BV were significantly lower in the RR+Iso group than in the RC and RR groups (Table 3, Fig. 4). Considering the suppression of bone density reduction by isoflavone intake, it was considered that remodeling of high turnover rotation was suppressed by isoflavone. Furthermore, it has been reported that subjects with high bone turnover markers had a high risk of fracture than low subjects in a meta-analysis that examined the relationship between bone turnover and bone strength (24). The low level of BFR, $\mathrm{mm}^{2} / \mathrm{mm}^{2} / \mathrm{y}, \mathrm{BFR} / \mathrm{BV}, \% \mathrm{y}$, $\mathrm{BFR} / \mathrm{TV}, \% \mathrm{y}$ in the RR+Iso group in this study may have higher bone strength compared to other groups.

The bone mass and body weight are closely related. This is because a person with a higher bone mass is subjected to higher mechanical load. Therefore, as body weight decreases, the bone mass decreases and the body weight becomes a predictor. A study of menopausal women reported that the bone mass decreases by $1-2 \%$ when the weight loss is $10 \%$ (25). In this study, the body weight and bone mass of the proximal region of the RR group decreased by $31.8 \%$ and $12.6 \%$ compared with those of the RC group (Fig. 1A, Fig. 3B). The weight loss and bone mass reduction were simultaneously observed, and it was considered that a part of bone loss in the RR group was due to lower mechanical load.

Like in previous studies $(5,13,14)$, no significant difference was found between the energy restricted group and the ad libitum feeding group for running distance in this study as well. Therefore, we considered that the effect of dietary restriction on bone mass reduction was stronger than that of running load in this study. We did not set a sedentary group, but wheel running and ad libitum feeding have been reported to increase BMD (26-28). By establishing the sedentary group, effect of both wheel running and energy restriction on bone can be evaluated.

There were some limitations to our study. First, because we did not have a sedentary control group, we could not make a comparison with a non-exercised group. The change in bone density of the RR group was not apparent compared with that of the non-exercise group to which energy restriction was applied. Second, we did not measure energy availability. As the LH level was not lowered, the present model was not similar to the hypothalamic amenorrhea model. Third, P40 used in this study is an isoflavone complex. Although it inhibited a decrease in bone mineral density, the effect of pure 
isoflavone could not be evaluated. Finally, as the number of sample was small, the result of this study is not very credible. In the future study, we will establish a sedentary energy-restricted group and confirm the position of the model of our study. Furthermore, evaluation of the effect of individual isoflavone is required in the future.

In summary, we examined the anti-osteoporotic effects of isoflavone on bone mineral density in female athlete rat model. Our results suggest that isoflavone intake inhibits bone loss caused by voluntary wheel running and energy restriction with decreased level of plasma $E_{2}$. Based on the results of bone morphometry analysis, we suggested that the mechanism of suppression of BMD reduction by isoflavone involves the suppression of high bone turnover induced by exercise and energy restriction. Our findings may suggest the possible novel role of isoflavone in osteoporosis among female athletes. This study provides basic data on the effect of isoflavone intake on osteoporosis of female athletes.

\section{Disclosure of State of COI}

The authors have no conflicts of interest directly relevant to the content of this article.

\section{Acknowledgments}

This study was supported in part by Global Center of Excellence (GCOE) Program of Japanese Ministry of Education, Culture, Sports and Technology.

\section{REFERENCES}

1) Otis CL, Drinkwater B, Johnson M, Loucks A, Wilmore J. 1997. American College of Sports Medicine position stand. The female athlete triad. Med Sci Sports Exerc 29(5): i-ix.

2) Ihle R, Loucks AB. 2004. Dose-response relationships between energy availability and bone turnover in young exercising women. J Bone Miner Res 19(8): 1231-1240.

3) Grinspoon S, Miller K, Coyle C, Krempin J, Armstrong C, Pitts S, Herzog D, Klibanski A. 1999. Severity of osteopenia in estrogen-deficient women with anorexia nervosa and hypothalamic amenorrhea. J Clin Endocrinol Metab 84(6): 2049-2055.

4) Williams NI, Helmreich DL, Parfitt DB, Caston-Balderrama A, Cameron JL. 2001. Evidence for a causal role of low energy availability in the induction of menstrual cycle disturbances during strenuous exercise training. J Clin Endocrinol Metab 86(11): 5184-5193.

5) Yanaka K, Higuchi M, Ishimi Y. 2012. Effect of longterm voluntary exercise and energy restriction on bone mineral density in mature female rats. J Phys Fitness Sports Med 1(4): 695-702.

6) Yanaka K, Takebayashi J, Matsumoto T, Ishimi Y. 2012. Determination of 15 isoflavone isomers in soy foods and supplements by high-performance liquid chromatography. J Agric Food Chem 60(16): 4012-4016.

7) da Graça R Campos M, Matos MP. 2010. Bioactivity of isoflavones: assessment through a theoretical model as a way to obtain a "Theoretical Efficacy Related to Estradiol (TERE)." Int J Mol Sci 11(2): 480-491.

8) Adlercreutz H, Hamalainen E, Gorbach S, Goldin B. 1992. Dietary phytoestrogens and the menopause in Japan. Lancet 339(8803): 1233.

9) Qi S, Zheng H. 2017. Combined effects of phytoestrogen genistein and silicon on ovariectomy-induced bone loss in rat. Biol Trace Elem Res 177(2): 281-287.

10) Zhang X, Shu XO, Li H, Yang G, Li Q, Gao YT, Zheng W. 2005. Prospective cohort study of soy food consumption and risk of bone fracture among postmenopausal women. Arch Intern Med 165(16): 1890-1895.

11) Wu J, Wang $X$, Chiba H, Higuchi M, Nakatani T, Ezaki O, Cui H, Yamada K, Ishimi Y. 2004. Combined intervention of soy isoflavone and moderate exercise prevents body fat elevation and bone loss in ovariectomized mice. Metabolism 53(7): 942-948.

12) Wu J, Oka J, Higuchi M, Tabata I, Toda T, Fujioka M, Fuku N, Teramoto T, Okuhira T, Ueno T, Uchiyama S, Urata K, Yamada K, Ishimi Y. 2006. Cooperative effects of isoflavones and exercise on bone and lipid metabolism in postmenopausal Japanese women: a randomized placebo-controlled trial. Metabolism 55(4): 423-433.

13) Aikawa Y, Agata U, Kakutani Y, Higano M, Hattori S, Ogata H, Ezawa I, Omi N. 2015. The interaction of voluntary running exercise and food restriction induces low bone strength and low bone mineral density in young female rats. Calcif Tissue Int 97(1): 90-99.

14) Aikawa Y, Agata U, Kakutani Y, Kato S, Noma Y, Hattori S, Ogata H, Ezawa I, Omi N. 2016. The preventive effect of calcium supplementation on weak bones caused by the interaction of exercise and food restriction in young female rats during the period from acquiring bone mass to maintaining bone mass. Calcif Tissue Int $\mathbf{9 8}(1)$ : 94-103.

15) Zhang Y, Li Q, Wan HY, Helferich WG, Wong MS. 2009. Genistein and a soy extract differentially affect threedimensional bone parameters and bone-specific gene expression in ovariectomized mice. J Nutr 139(12): 2230-2236.

16) Sehmisch S, Erren M, Kolios L, Tezval M, SeidlovaWuttke D, Wuttke W, Stuermer KM, Stuermer EK. 2010. Effects of isoflavones equol and genistein on bone quality in a rat osteopenia model. Phytother Res 2: S168-174.

17) Miao Q, Li JG, Miao S, Hu N, Zhang J, Zhang S, Xie YH, Wang JB, Wang SW. 2012. The bone-protective effect of genistein in the animal model of bilateral ovariectomy: roles of phytoestrogens and PTH/PTHR1 against postmenopausal osteoporosis. Int J Mol Sci 13(1): 56-70.

18) Tousen Y, Wolber FM, Chua WH, Tadaishi M, Ishimi Y, Kruger MC. 2014. Effects of daidzein and kiwifruit on bone mineral density and equol production in ovariectomised rats. Int J Food Sci Nutr 65(3): 360-367.

19) Hidaka S, Okamoto Y, Miyazaki K, Uesugi T. 2003. Evaluation of a soybean product fujiflavone $\mathrm{P} 40$ as an antiosteoporotic agent in rats. Phytother Res 17(2): 112-119.

20) Loucks AB. 2006. The response of luteinizing hormone pulsatility to 5 days of low energy availability disappears by 14 years of gynecological age. J Clin Endocrinol Metab 91(8): 3158-3164.

21) Williams NI, Young JC, McArthur JW, Bullen B, Skrinar GS, Turnbull B. 1995. Strenuous exercise with caloric restriction: effect on luteinizing hormone secretion. Med Sci Sports Exerc 27(10): 1390-1398.

22) Dimarco NM, Dart L, Sanborn CB. 2007. Modified activity-stress paradigm in an animal model of the female athlete triad. J Appl Physiol 103(5): 1469-1478.

23) Wang G. 1923. The relation between "spontaneous" activity and oestrous cycle in the white rat. Comp Psychol Monogr 2: 1-27. 
24) Johnell O, Odén A, De Laet C, Garnero P, Delmas PD, Kanis JA. 2002. Biochemical indices of bone turnover and the assessment of fracture probability. Osteoporos Int 13(7): 523-526.

25) Shapses SA, Riedt CS. 2006. Bone, body weight, and weight reduction: what are the concerns? J Nutr 136(6): 1453-1456.

26) Omi N, Morikawa N, Ezawa I. 1994. The effect of voluntary exercise on bone mineral density and skeletal muscles in the rat model at ovariectomized and sham stages.
Bone Miner 24(3): 211-222.

27) Shiga K, Hara H, Okano G, Ito M, Minami A, Tomita F. 2003. Ingestion of difructose anhydride III and voluntary running exercise independently increase femoral and tibial bone mineral density and bone strength with increasing calcium absorption in rats. J Nutr 133(12): 4207-4211.

28) Holy X, Zérath E. 2000. Bone mass increases in less than 4 wk of voluntary exercising in growing rats. Med Sci Sports Exerc 32(9): 1562-1569. 